Linköping Studies in Science and Technology.

Licentiate Thesis No. 1632

\title{
Composite Structure Optimization using a Homogenized Material Approach
}

\section{Dženan Hozić}

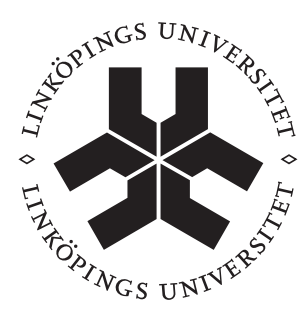

\section{Linköping University INSTITUTE OF TECHNOLOGY}

Department of Management and Engineering, Division of Mechanics Linköping University, SE-581 83, Linköping, Sweden 
Printed by:

LiU-Tryck, Linköping, Sweden, 2013

ISBN 978-91-7519-453-0

ISSN 0280-7971

Distributed by:

Linköping University

Department of Management and Engineering

SE-581 83, Linköping, Sweden

\section{(C) 2013 Dženan Hozić}

This document was prepared with ATEX, December 2, 2013

No part of this publication may be reproduced, stored in a retrieval system, or be transmitted, in any form or by any means, electronic, mechanical, photocopying, recording, or otherwise, without prior permission of the author. 


\section{Preface}

This licentiate thesis has been funded by the Swedish Foundation for Strategic Research (SSF) through the ProViking Program.

First and foremost I would like to express my gratitude to my supervisors Anders Klarbring and Bo Torsenfeldt for the guidance and support they have provided during my work on this thesis. Furthermore, I would also like to thank all my colleagues at the Division of Mechanics for their support and help, and for making my time at the division pleasant and interesting.

Most importantly I would like to thank my family for all their support, especially my wonderful and loving wife Azra for all the love, support and understanding she has given me.

\section{Dženan Hozić}

Linköping, December 2013 



\section{Abstract}

The increasing use of fibre-reinforced composite materials in the manufacturing of high performance structures is primarily driven by their superior strength-toweight ratio when compared to traditional metallic alloys. This provides the ability to design and manufacture lighter structures with improved mechanical properties. However, the specific manufacturing process of composite structures, along with the orthotropic material properties exhibited by fibre-reinforced composite materials, result in a complex structural design process where a number of different design parameters and manufacturing issues, which affect the mechanical properties of the composite structure, have to be considered. An efficient way to do this is to implement structural optimization techniques in the structural design process thus improving the ability of the design process to find design solutions which satisfy the structural requirements imposed on the composite structure.

This thesis describes a two phase composite structure optimization method based on a novel material homogenization approach. The proposed method consists of a stiffness optimization problem and a lay-up optimization problem, respectively, with the aim to obtain a manufacturable composite structure with maximized stiffness properties. The homogenization material approach is applied in both optimization problems, such that the material properties of the composite structure are homogenized. In the proposed method the stiffness optimization problem provides a composite structure with maximized stiffness properties by finding the optimal distribution of composite material across the design domain. The aim of the lay-up optimization problem is to obtain a manufacturable lay-up sequence of fibre-reinforced composite plies for the composite structure which, as far as possible, retains the stiffness properties given by the stiffness optimization problem. The ability of the composite structure optimization method to obtain manufacturable composite structures is tested and confirmed by a number of numerical tests. 



\section{List of Papers}

The following papers is included in thesis:

I. Dž. Hozić, A. Klarbring, B. Torstenfelt. (2013). Density Filter Control of Thickness-to-Length Change of Composite Structures. Published in proceeding of $10^{\text {th }}$ World Congress on Structural and Multidisciplinary Optimization.

II. Dž. Hozić, A. Klarbring, B. Torstenfelt. (2013). Stiffness and Lay-up Optimization of Composite Structures based on a Homogenized Material Approach. Submitted. 



\section{Contents}

Preface iii

Abstract $\quad$ V

List of Papers vii

$\begin{array}{ll}\text { Contents } & \text { ix }\end{array}$

Part I - Introduction and Background 1

1 Introduction 3

2 Structural Optimization $\quad 5$

2.1 General Formulation . . . . . . . . . . . . . . . . 5

2.2 Solution Methods . . . . . . . . . . . . . . . . 6

2.3 Types of Structural Optimization Problems . . . . . . . . . . 8

3 Composites $\quad 9$

3.1 Composite Materials . . . . . . . . . . . . . . . . . . 9

3.1.1 Material Properties . . . . . . . . . . . . . . . . . . 9

3.2 Composite Structures . . . . . . . . . . . . . . . . . . . 11

3.2.1 Constitutive Equations . . . . . . . . . . . . . . . . 12

3.2 .2 Mechanical Properties . . . . . . . . . . . . . . . . . 14

$\begin{array}{lll}4 & \text { Review of included papers } & 17\end{array}$

$\begin{array}{lr}\text { Bibliography } & 19\end{array}$

Part II - Included papers 21

Paper I:Density Filter Control of Thickness-to-Length Change of Composite Structures . . . . . . . . . . . . . . . 25

Paper II: Stiffness and Lay-up Optimization of Composite Structures based on a Homogenized Material Approach . . . . . . . . . . . . 37 



\section{Part I}

\section{Introduction and Background}





\section{Introduction}

For the last two decades composite materials have increasingly substituted metallic alloys in the manufacturing of high performance structures. The main driving factor behind such a development is the superior strength-to-weight ratio exhibited by composite materials when compared to metallic alloys. This allows for the manufacturing of lighter structures with improved mechanical properties, such as stiffness and strength. The most commonly applied type of composite materials is unidirectional (UD) fibre-reinforced composite plies, which have orthotropic material properties. To manufacture, a composite structure requires the UD plies to be stacked in a predefined lay-up sequence to form the composite structure. This specific manufacturing process together with the orthotropic material properties of the UD plies provide the designers with the ability to tailor the mechanical behaviour of a composite structure. However, this also results in a complex structural design process where a number of design parameters affecting the mechanical properties of a composite structures have to be considered. Parameters that primarily affect the mechanical properties of a composite structure are (i) the fibre orientation $\theta$ of the fibrous constituents of the UD plies, (ii) the total amount of material with a given fibre orientation $\theta$ used to manufacture the composite structure, (iii) the internal order of the UD plies within the lay-up sequence of the structure. Apart from these design parameters, consideration must be given to manufacturing issues in the form of stacking sequence rules in order to minimize effects that can lead to degradation of the mechanical properties of the composite structure. The number of design solutions that can be generated by such a structural design process is large, which makes it difficult to find a solution that best fulfills the desired structural requirements imposed onto the composite structure. Implementing structural optimization techniques in the design process of composite structures will provide the ability to formulate structural optimization problems, where design parameters and manufacturing issues influencing the mechanical properties of a composite structure can be efficiently taken into account, and thereby improving the ability of the structural design process to find design solutions which satisfy the structural requirement imposed on the composite structure.

In light of this, we have formulated a composite structure optimization method which is based on a novel homogenization approach for parametrization and modelling of composite material in structural optimization problems. The proposed composite structure optimization method consists of two phases which contain a 
stiffness optimization problem and a lay-up optimization problem, respectively, which are solved in sequence. The method assumes that the composite structure only contains composite material with fibre orientations $\theta$ from a predefined set of angles $\Theta$.

In the stiffness optimization problem the homogenized material approach is applied so that the material properties associated to each fibre orientation $\theta \in \Theta$ are uniformly distributed across the entire structure. This results in a homogenization of material properties of the structure, which enables the problem formulation to be treated in a similar fashion to a variable thickness sheet problem, see Christensen and Klarbring (2008), thus allowing the stiffness optimization problem to be efficiently solved by gradient-based optimization algorithms. In the problem formulation design constraints are set on the total volume of the composite structure and on how much composite material of each fibre orientation $\theta \in \Theta$ is allowed to be used. The design variables of the problem formulation represent the total amount of material of each fibre orientation $\theta \in \Theta$. The aim of the stiffness optimization problem is to maximize the stiffness of the structure by finding the optimal distribution of composite material across the entire design domain.

In the second phase of the proposed method, the lay-up optimization problem aims to obtain a manufacturable lay-up sequence of UD plies such that the stiffness properties of the composite structure obtained by the stiffness optimization problem are, as far as possible, maintained. The objective function of the lay-up optimization problem is to minimize the least squares difference between a set of target value lamination parameters and a set of discrete based lamination parameters, see Tsai and Hahn (1980). The sets of lamination parameters are here treated as stiffness measures for the composite structure. The homogenized material approach is again applied to calculate the set of target value lamination parameters from the results obtained by the stiffness optimization problem. This gives a direct measure of the stiffness properties obtained by the stiffness optimization problem. The design variables of the lay-up optimization problem are a set of binary design variables for each UD ply in the lay-up sequence, where each design variable represents one of the fibre orientations $\theta \in \Theta$. The constraints that are applied in the lay-up optimization problem represent stacking sequence rules which aim take into consideration some of the manufacturing issues of composite structures. The layup optimization problem is a mixed integer programming problem and is efficiently solved using a branch-and-cut optimization algorithm, see Wolsey (1998). 


\section{Structural Optimization}

\subsection{General Formulation}

Structural optimization is a subset of the field of mathematical design optimization, which is focused on mechanical structures whose main task is to carry applied loads. In general, structural optimization is the subject of obtaining an assemblage of material, a structure, which sustains the applied loads in the best possible way. Applying mathematical design optimization methods provides the ability to formulate structural optimization problems where requirements on the structural performance, i.e. objectives and constraints, are given precise mathematical form. Typical measures of performance in structural optimization problems are weight, stiffness, critical load, stress, displacement and geometrical parameters. The structural optimization problems are formulated by choosing one of the above structural performance measures as the objective function and one or more of the remaining ones as constraints in the problem formulation, see Christensen and Klarbring (2008) and Bendsøe and Sigmund (2003) for general overviews.

The general form of a structural optimization problem is as follows:

$$
(\mathrm{SO})\left\{\begin{array}{l}
\min _{\mathbf{x}, \mathbf{y}} f(\mathbf{x}, \mathbf{y}) \\
\text { subject to }\left\{\begin{array}{l}
\mathbf{g}_{\mathrm{E}}(\mathbf{x}, \mathbf{y})=\mathbf{0} \\
\mathbf{g}_{\mathrm{B}}(\mathbf{y}) \leq \mathbf{0} \\
\mathbf{g}_{\mathrm{D}}(\mathbf{x}) \leq \mathbf{0},
\end{array}\right.
\end{array}\right.
$$

where the objective function $f(\mathbf{x}, \mathbf{y})$ is a measure which indicates how well the current design achieves the desired objective of the optimization. Typically the choice of objective function is such that a minimization problem is obtained, i.e. decreasing values of the objective function result in better solutions. The design variable vector $\mathbf{x}$, describes the current design of the structure. In structural optimization the design variables often represent geometrical parameters such as area of a bar and thickness of a sheet, or material choice parameters like fibre orientations in composite plies or material densities. The state variable vector $\mathbf{y}$, represents the response of the structure, i.e. it represent the current state of the structure. To be more precise it often represents displacements or stresses. Constraints that are applied to the (SO) problem are typically divided into three types: 
- The equilibrium constraint $\mathbf{g}_{\mathrm{E}}(\mathbf{x}, \mathbf{y})=\mathbf{0}$ is used to calculate the response of the structure at a given design point $\mathbf{x}$. For a discretized structural optimization problem the equilibrium constraint has the form

$$
\mathbf{K}(\mathbf{x}) \mathbf{u}=\mathbf{F}(\mathbf{x}),
$$

where the $\mathbf{K}(\mathbf{x})$ is the stiffness matrix of the structure and is generally a function of the design variable $\mathbf{x} ; \mathbf{F}(\mathbf{x})$ is the load vector applied on the structure, which also can be dependent on $\mathbf{x}$ and $\mathbf{u}$ is the displacement vector which is treated as the state variable $\mathbf{y}$ in the problem formulation.

- The behavioural constraints $\mathbf{g}_{\mathrm{B}}(\mathbf{y}) \leq 0$ put limitations on the state variable y.

- The design constraints $\mathbf{g}_{\mathrm{D}}(\mathbf{x}) \leq 0$ put constraints on the design variable $\mathbf{x}$.

Problem (SO) is a simultaneous formulation of a structural optimization problem, where the state variable $\mathbf{y}$ and the design variable $\mathbf{x}$ are treated as independent variables. However, a common situation in structural optimization is that the state problem uniquely defines the state variable $\mathbf{y}$ for each given design point $\mathbf{x}$. In these cases we get the following from the equilibrium constraint (1), if $\mathbf{K}(\mathbf{x})$ is non-singular

$$
\mathbf{u}=\mathbf{K}(\mathbf{x})^{-1} \mathbf{F}(\mathbf{x})
$$

indicating that the state variable vector $\mathbf{u}$ can be expressed as a function of the design variable vector $\mathbf{x}$ rather then be treated as an independent variable vector, i.e. $\mathbf{y}=\mathbf{u}=\mathbf{u}(\mathbf{x})$. With this conclusion in mind, a reformulation of (SO) can be performed by removing the equilibrium constraint $\mathbf{g}_{\mathrm{E}}(\mathbf{x}, \mathbf{y})=0$ from the problem formulation, thus giving way to a nested formulation of the structural optimization problem according to

$$
(\mathrm{SO})_{\mathrm{nf}}\left\{\begin{array}{l}
\min _{\mathbf{x}} f(\mathbf{x}, \mathbf{u}(\mathbf{x})) \\
\text { subject to } \quad \mathbf{g}(\mathbf{x}, \mathbf{u}(\mathbf{x})) \leq \mathbf{0} .
\end{array}\right.
$$

In the nested formulation of the structural optimization problem the only variables are given by the design variable vector $\mathbf{x}$, while the state variable vector $\mathbf{u}(\mathbf{x})$ is given implicitly by separately solving the equilibrium constraint. The constraint function $\mathbf{g}(\mathbf{x}, \mathbf{u}(\mathbf{x})) \leq \mathbf{0}$ now represents all the behavioural and design constraints applied in the problem formulation. The nested formulation $(\mathrm{SO})_{\mathrm{nf}}$ is usually the starting point when applying numerical method to generate solutions to a structural optimization problem.

\subsection{Solution Methods}

In general, there are two main obstacles when solving structural optimization problems. The first one is that most problems tend to be nonconvex and thus are diffi- 
cult to solve. The second obstacle is the fact that objective and constraint functions are very difficult to obtain as explicit functions of the design variables for larger nested structural optimization problems. A way to circumvent these issues when solving larger problems is to generate a sequence of explicit subproblems which are then solved instead of the original problem. These subproblems are approximations of the original problem and are usually chosen such that they are convex.

There are a number of different methods frequently used to generate explicit convex approximations, i.e. subproblems $(\mathrm{SO})_{\mathrm{nf}}^{k}$, of the original nested problem formulation (SO) nf. Some of these include the Sequential Linear Programming (SLP) and Sequential Quadratic Programming (SQP) approximations, which are designed to solve general nonlinear optimization problems. SLP and SQP use Taylor expansion to linearize the objective and constraint functions at a design point $\mathbf{x}^{k}$, which generate explicit convex approximations of $(\mathrm{SO})_{\mathrm{nf}}$ at $\mathrm{x}^{k}$. Other methods, such as Convex Linearization (CONLIN), Optimality Criteria (OC) and The Method of Moving Asymptotes (MMA) developed by Svanberg (1987), generate convex subproblems to $(\mathrm{SO})_{\mathrm{nf}}$ by linearizing in such a way that special characteristics of the structural optimization problem can be taken into account. As a result, the subproblems generated by CONLIN, OC and MMA methods tend to be more exact approximations of $(\mathrm{SO})_{\mathrm{nf}}$ then the approximations generated by SLP and SQP. All of the approximation method above for generating explicit convex subproblems $(\mathrm{SO})_{\mathrm{nf}}^{k}$ are gradient based methods which are the most common type of optimization methods used to solve structural optimization problems. These methods require, at each design point $\mathrm{x}^{k}$, information about the objective and constraint functions and their respective derivatives in order to solve the subproblems.

Although most structural optimization problems are solved using gradient based methods, some problem formulations can have certain characteristics which make gradient based methods generally unsuitable. One such example is structural optimization of composite structure, where the problem formulation often has discrete design variables. This makes it difficult to obtain the necessary derivatives of the objective and constraint functions which are required for gradient based optimization methods. For such cases, zero order optimization methods are more applicable as they do not require any gradient information from the objective and constraint functions. However, they are generally not as efficient and robust as gradient based optimization methods. For this reason we have in the present work formulated a two phase composite structure optimization method. It consists of two structural optimization problems for composite structures, where a gradient based and a zero order optimization method are used to optimize the composite structure. This allows us to make use of the more efficient and robust gradient based optimization method while also retaining the unique characteristics of composite structures. 


\subsection{Types of Structural Optimization Problems}

Structural optimization problems are usually divided into three types of problem formulations. The classification is done according to what the design variable $\mathbf{x}$ represents in the structural optimization problem. The three types of structural optimization problems are shape optimization, sizing optimization and topology optimization and they all address different aspects of structural design optimization.

In shape optimization the goal is to find the optimal shape of a structure. This is done by allowing parts of the boundary of the design domain to change during the optimization. The parts of the boundary allowed to change are usually represented by polynomial functions where the design variables are vertices controlling the shape of the polynomials. Any change in the position of the vertices will result in a changed shape of the polynomial functions, thus changing the shape of the structure.

In sizing optimization problems the design domain is known and fixed throughout the optimization. Sizing optimization typically treats 2-D continuum structures like plates or discrete structure like a truss structure. The goal of sizing optimization is to find optimal thickness distributions of plates, or in case of truss structures, to find the optimal cross-sectional areas of the truss members. In these problem formulations the design variables typically represent the thickness of the structure or cross-sectional areas of truss members.

Topology optimization is considered the most general form of structural design optimization. In essence the goal with topology optimization is to find the optimal distribution of material within a design domain, such that each point of the design domain is allowed to either have full material or no material. To achieve this topological feature a penalization is introduced on the design variables so that they only take on two value, 0 for no material and a fixed upper bound value for full material. The design variables in topology optimization also represent crosssectional areas of truss members and thickness of a 2-D structure. This formulation can also be extended to 3-D structures where the design variable is taken to be a density-like variable which can only take the value 0 for no material and 1 for full material. 


\section{Composites}

\subsection{Composite Materials}

Composite materials are formed by combining two or more materials, known as constituents. The characteristics of a composite material, such as physical and mechanical properties, are dependent on how the constituents of the composite material are assembled. In general, composite materials retain the combined characteristics of the constituents, but in some cases the characteristics of the composite material can surpass or be substantially different from those of its individual constituents. Composite materials are in turn classified into a number of different types, based on the shape of its constituents. However, in the present work we are only considering composite material of the type fibre-reinforced composite material, which is the most common type of composite material used for manufacturing high performance structures and structural components. Fibre-reinforced composite material is generally made up of two constituents. A fibrous constituent which has high strength and stiffness properties and as such is the main load-carrying constituent. The second constituent is a matrix material which the fibrous constituents are embedded within. The main task of the matrix constituent is to hold the fibrous constituents together, act as a load-transferring medium and also to protect the fibrous constituents from environmental effects. Togheter the constituents form a sheet of fibre-reinforced composite material, a ply, see Reddy (2004), Campbell (2010) and Gürdal et al. (1999)

\subsubsection{Material Properties}

The material properties of a fibre-reinforced composite ply is primarily dependent on what kind of fibrous constituent is used. In the present work, only continuous unidirectional (UD) fibrous constituents are considered. 


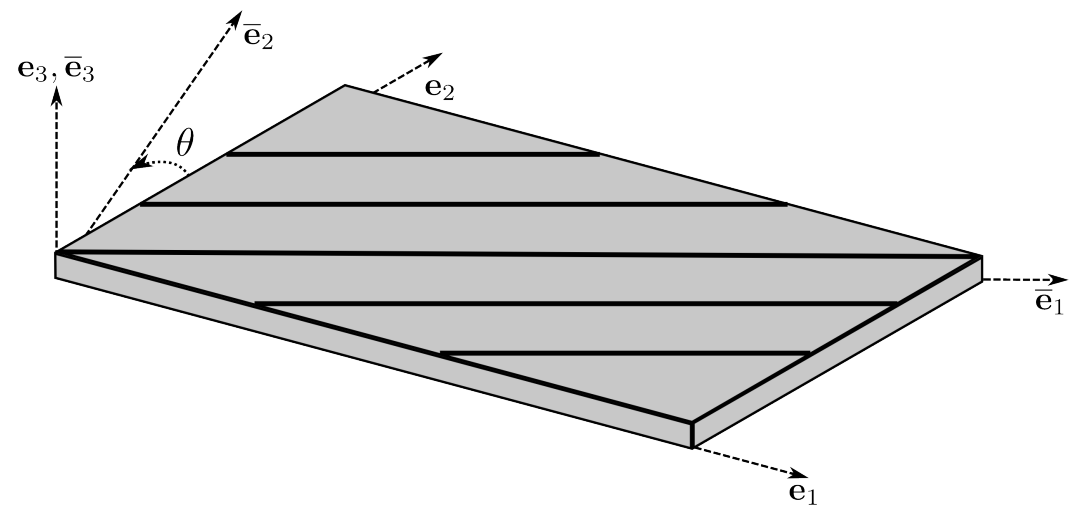

Figure 1: UD fibre-reinforced composite ply

As figure 1 illustrates, in this type of composite material all of the fibrous constituents are aligned in the same direction and run the entire length of a ply. The fibrous constituents exhibit high strength and stiffness properties in the direction parallel to the fibres but in the transversal directions the corresponding properties are substantially weaker. For this reason the UD fibre-reinforced composite plies are said to exhibit orthotropic material properties, as the material properties of the ply change with the orientation of the fibrous constituents.

\section{Constitutive Relation}

A typical fibre-reinforced composite ply is thin in comparison with its other dimensions and experiences a plane state of stress. For such a case the stress-strain relationship is given in the fixed global $\left(\mathbf{e}_{1}, \mathbf{e}_{2}, \mathbf{e}_{3}\right)$-frame as

$$
\left\{\begin{array}{l}
\sigma_{1} \\
\sigma_{2} \\
\sigma_{4} \\
\sigma_{5} \\
\sigma_{6}
\end{array}\right\}=\left[\begin{array}{ccccc}
Q_{11} & Q_{12} & Q_{14} & 0 & 0 \\
Q_{12} & Q_{22} & Q_{24} & 0 & 0 \\
Q_{14} & Q_{24} & Q_{44} & 0 & 0 \\
0 & 0 & 0 & Q_{55} & Q_{56} \\
0 & 0 & 0 & Q_{56} & Q_{66}
\end{array}\right]\left\{\begin{array}{l}
\varepsilon_{1} \\
\varepsilon_{2} \\
\varepsilon_{4} \\
\varepsilon_{5} \\
\varepsilon_{6}
\end{array}\right\} .
$$

In equation (2) $\sigma_{j}$ and $\varepsilon_{j}$ represent the stress and strain components, respectively. The strain components $\varepsilon_{j}$ above are given as

$$
\left\{\begin{array}{l}
\varepsilon_{1} \\
\varepsilon_{2} \\
\varepsilon_{4} \\
\varepsilon_{5} \\
\varepsilon_{6}
\end{array}\right\}=\left\{\begin{array}{l}
\varepsilon_{1}^{\text {mem }} \\
\varepsilon_{2}^{\text {mem }} \\
\varepsilon_{4}^{\text {mem }} \\
\varepsilon_{5}^{\text {mem }} \\
\varepsilon_{6}^{\text {mem }}
\end{array}\right\}+z\left\{\begin{array}{l}
\varepsilon_{1}^{\text {cur }} \\
\varepsilon_{2}^{\text {cur }} \\
\varepsilon_{4}^{\text {cur }} \\
\varepsilon_{5}^{\text {cur }} \\
\varepsilon_{6}^{\text {cur }}
\end{array}\right\}
$$

where $\varepsilon_{j}^{\text {mem }}$ and $\varepsilon_{j}^{\text {cur }}$ are the mid-plane strains and bending strains, respectively, and $z$ is a thickness variable, see Reddy (2004). Furthermore, $Q_{i j}$ in equation (2) 
are the elements of the material coefficient matrix $\mathbf{Q}$ of a ply, expressed in the global $\left(\mathbf{e}_{1}, \mathbf{e}_{2}, \mathbf{e}_{3}\right)$-frame which is given according to

$$
\mathrm{Q}=\mathbf{T}^{T} \mathbf{C T} .
$$

In equation (4), $\mathbf{C}$ is the material coefficient matrix expressed in the material $\left(\overline{\mathbf{e}}_{1}, \overline{\mathbf{e}}_{2}, \overline{\mathbf{e}}_{3}\right)$-frame which follows the orientation of the fibrous constituents in the $\mathbf{e}_{1} \mathbf{e}_{2}$-plane as shown in figure 1. The elements of $\mathbf{C}$ are expressed in terms of engineering constants as

$$
\mathbf{C}=\left[\begin{array}{ccccc}
\frac{E_{1}}{1-\nu_{12} \nu_{12}} & \frac{\nu_{12} E_{2}}{1-\nu_{12} \nu_{12}} & 0 & 0 & 0 \\
\frac{\nu_{12} E_{2}}{1-\nu_{12} \nu_{12}} & \frac{E_{2}}{1-\nu_{12} \nu_{12}} & 0 & 0 & 0 \\
0 & 0 & G_{12} & 0 & 0 \\
0 & 0 & 0 & G_{23} & 0 \\
0 & 0 & 0 & 0 & G_{13}
\end{array}\right]
$$

where $E_{1}, E_{2}$ are the Young's modulus, $G_{12}, G_{23}, G_{13}$ represent the shear modulus and $\nu_{12}, \nu_{21}$ are the Poisson's ratios of a UD fibre-reinforced ply, see Reddy (2004). The transformation matrix $\mathbf{T}$ in equation (4) represents a transformation from the material $\left(\overline{\mathbf{e}}_{1}, \overline{\mathbf{e}}_{2}, \overline{\mathbf{e}}_{3}\right)$-frame to the global $\left(\mathbf{e}_{1}, \mathbf{e}_{2}, \mathbf{e}_{3}\right)$-frame, see Cook et al. (2003), as

$$
\mathbf{T}=\left[\begin{array}{ccccc}
\cos ^{2} \theta & \sin ^{2} \theta & \cos \theta \sin \theta & 0 & 0 \\
\sin ^{2} \theta & \cos ^{2} \theta & -\cos \theta \sin \theta & 0 & 0 \\
-2 \cos \theta \sin \theta & 2 \cos \theta \sin \theta & \cos ^{2} \theta-\sin ^{2} \theta & 0 & 0 \\
0 & 0 & 0 & \cos \theta & -\sin \theta \\
0 & 0 & 0 & \sin \theta & \cos \theta
\end{array}\right]
$$

where $\theta$ is an arbitrary angle given by a counter-clockwise sense rotation of the material $\left(\overline{\mathbf{e}}_{1}, \overline{\mathbf{e}}_{2}, \overline{\mathbf{e}}_{3}\right)$-frame about the transversal normal direction $\mathbf{e}_{3}$ as shown in figure 1 and is usually referred to as the fibre orientation $\theta$ of a UD fibre-reinforced composite ply.

\subsection{Composite Structures}

A composite structure is manufactured by stacking UD fibre-reinforced plies to form the structure. The orthotropic material properties of the plies provide the ability to tailor the mechanical properties of the composite structure by orienting the plies so the fibrous constituents are oriented in a number of different fibre orientations $\theta$, as illustrated in figure 2 . 


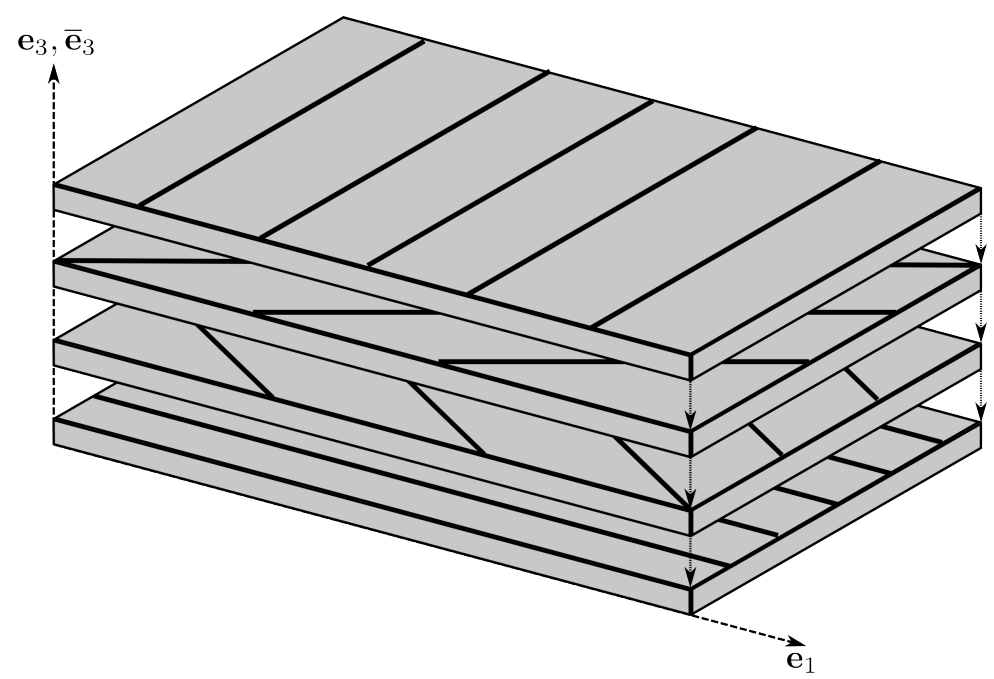

Figure 2: Illustration of composite structure

In general the composite plies can be oriented in such a way so the fibre orientations $\theta$ of the plies can take on any angle between $0^{\circ}$ and $180^{\circ}$. However, when manufacturing composite structures the fibre orientations $\theta$ are restricted to a fixed set of angles. The most frequently used set of angles is

$$
\Theta=\left\{0^{\circ}, \pm 45^{\circ}, 90^{\circ}\right\}
$$

but for some applications $\Theta$ is complemented with $\pm 30^{\circ}$ and $\pm 60^{\circ}$ angles or they can also replace the $\pm 45^{\circ}$ angles of the set. Plies with fibre orientations $0^{\circ}$ and $90^{\circ}$ are known as on-axis plies while those with fibre orientations $\pm \theta$ are known as off-axis plies. The internal order in which plies with fibre orientations $\theta \in \Theta$ are stacked to form the composite structure is known as a lay-up sequence, which describes what mechanical properties are retained by the composite structure.

\subsubsection{Constitutive Equations}

For a composite structure which is modelled according to first order shear deformation theory and has a general lay-up sequence of UD fibre-reinforced composite plies, the governing constitutive equations are given, see Reddy (2004), as

$$
\left\{\begin{array}{l}
N_{11} \\
N_{22} \\
N_{12} \\
M_{11} \\
M_{22} \\
M_{12} \\
T_{2} \\
T_{1}
\end{array}\right\}=\left[\begin{array}{cccccccc}
A_{11} & A_{12} & A_{14} & B_{11} & B_{12} & B_{14} & 0 & 0 \\
A_{12} & A_{22} & A_{24} & B_{12} & B_{22} & B_{24} & 0 & 0 \\
A_{14} & A_{24} & A_{44} & B_{14} & B_{24} & B_{44} & 0 & 0 \\
B_{11} & B_{12} & B_{14} & D_{11} & D_{12} & B_{14} & 0 & 0 \\
B_{12} & B_{22} & B_{24} & D_{12} & D_{22} & B_{24} & 0 & 0 \\
B_{14} & B_{24} & B_{44} & D_{14} & D_{24} & D_{44} & 0 & 0 \\
0 & 0 & 0 & 0 & 0 & 0 & \tilde{A}_{55} & \tilde{A}_{56} \\
0 & 0 & 0 & 0 & 0 & 0 & \tilde{A}_{56} & \tilde{A}_{66}
\end{array}\right]\left\{\begin{array}{l}
\varepsilon_{1}^{\text {mem }} \\
\varepsilon_{2}^{\text {mem }} \\
\varepsilon_{4}^{\text {mem }} \\
\varepsilon_{1}^{\text {cur }} \\
\varepsilon_{2}^{\text {cur }} \\
\varepsilon_{4}^{\text {cur }} \\
\varepsilon_{5}^{\text {mem }} \\
\varepsilon_{6}^{\text {mem }}
\end{array}\right\},
$$


where $N_{i j}$ represent the in-plane force resultants, $M_{i j}$ are the moment resultants and $T_{i}$ are the transversal shear force resultants of the composite structure. The submatrices in equation (7) correspond to the extensional $\mathbf{A}$, the bending-extensional coupling $\mathbf{B}$, the bending $\mathbf{D}$ and the transversal shear $\tilde{\mathbf{A}}$ stiffness matrices, respectively. For a general lay-up sequence of an even number $n$ UD plies as shown in figure 3, the stiffness submatrices in equation (7) are expressed, see Tsai and Hahn (1980) and Reddy (2004), as

$$
\begin{aligned}
& A_{i j}=\sum_{k=1-n / 2}^{n / 2}\left(\int_{z_{k-1}}^{z_{k}} Q_{i j}^{k} d z\right) \quad i, j=1,2,4, \\
& \tilde{A}_{l m}=\sum_{k=1-n / 2}^{n / 2}\left(\int_{z_{k-1}}^{z_{k}} K_{s} Q_{l m}^{k} d z\right) \quad l, m=5,6, \\
& B_{i j}=\sum_{k=1-n / 2}^{n / 2}\left(\int_{z_{k-1}}^{z_{k}} Q_{i j}^{k} z d z\right) \quad i, j=1,2,4, \\
& D_{i j}=\sum_{k=1-n / 2}^{n / 2}\left(\int_{z_{k-1}}^{z_{k}} Q_{i j}^{k} z^{2} d z\right) \quad i, j=1,2,4 .
\end{aligned}
$$

where $k$ is the ordinal number of a ply, $K_{s}$ is the shear correlation factor and $z_{k}$ marks the position of the $k$-th ply in the lay-up sequence, along the thickness of the structure. For each ply $k$ in the lay-up sequence, $Q_{i j}^{k}$ and $Q_{l m}^{k}$ in equations (8a)-(8d) represent the elements of the material coefficient matrix $\mathbf{Q}$ as given by equation (4) for a fixed fibre orientation $\theta_{k} \in \Theta$.

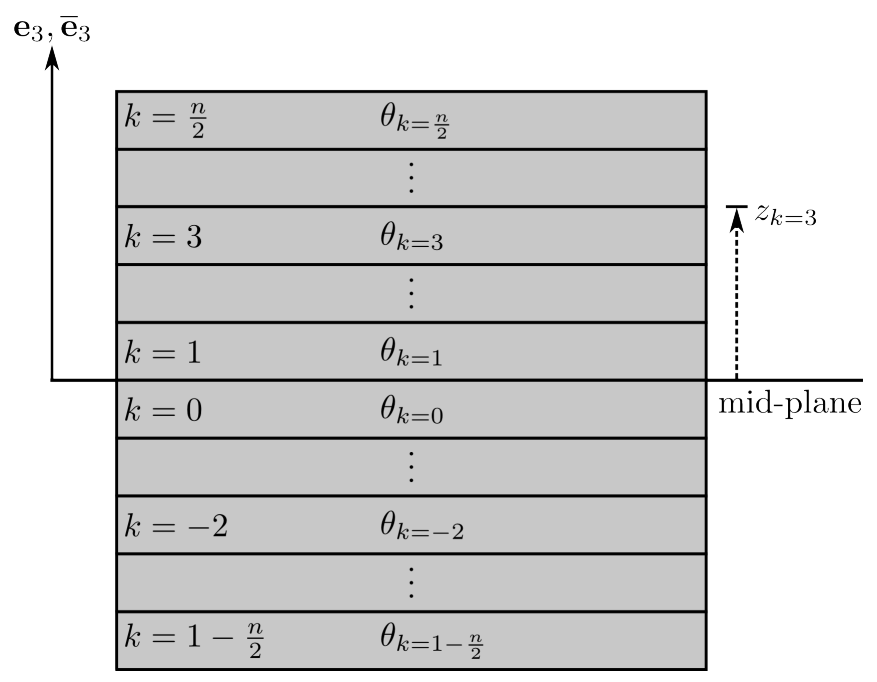

Figure 3: A general lay-up sequence of UD fibre-reinforced plies 
Each ply $k$ in the lay-up sequence in figure 3 can be assumed to have a uniform and equal thickness $h_{p l y}$, hence the position of a ply in the lay-up sequence can be given as

$$
z_{k}=k h_{p l y}
$$

which inserted into into equations (8a)-(8d) allows the stiffness matrices to be expressed according to

$$
\begin{aligned}
& A_{i j}=h_{p l y} \sum_{k=1-n / 2}^{n / 2}[k-(k-1)] Q_{i j}^{k} \quad i, j=1,2,4, \\
& \tilde{A}_{l m}=h_{p l y} K_{s} \sum_{k=1-n / 2}^{n / 2}[k-(k-1)] Q_{l m}^{k} \quad l, m=5,6, \\
& B_{i j}=\frac{h_{p l y}^{2}}{2} \sum_{k=1-n / 2}^{n / 2}\left[k^{2}-(k-1)^{2}\right] Q_{i j}^{k} \quad i, j=1,2,4, \\
& D_{i j}=\frac{h_{p l y}^{3}}{3} \sum_{k=1-n / 2}^{n / 2}\left[k^{3}-(k-1)^{3}\right] Q_{i j}^{k} \quad i, j=1,2,4,
\end{aligned}
$$

\subsubsection{Mechanical Properties}

The constitutive equations in equation (7), together with the stiffness matrices expressed in equations (9a)-(9d), are sufficient to describe the mechanical behaviour of a composite structure which is subjected to any kind of in-plane, shear or bending load case, or any combination of these load cases. From equations (9a) and (9b) it can be observed that the extensional and transversal shear stiffness properties of a composite structure are independent of the lay-up sequence of the plies. They are only a function of the total amount of plies with different fibre orientations $\theta \in \Theta$, used in the composite structure. The reason for this lies in the fact that the weight factors within the brackets in equations (9a) and (9b) retain a unity value for every ply regardless of its position within the lay-up sequence of the composite structure. This however is not true for the coupling stiffness matrix $\mathbf{B}$ and bending stiffness matrix $\mathbf{D}$ of the composite structure expressed in equations (9c) and (9d), respectively. Here the weight factor values of a ply increase as its position in the lay-up sequence is moved from the mid-plane of the composite structure. This implies that plies located further away from the mid-plane in the lay-up sequence will have a larger contribution to the coupling and bending stiffness properties of the composite structure. The importance of the internal ordering of the plies within the lay-up sequence is more pronounced for the bending stiffness matrix $\mathbf{D}$ in equation (9d) then for the coupling stiffness matrix B in equation (9c). Also, the weight factor values for the coupling stiffness matrix $\mathbf{B}$ are antisymmetric with 
respect to the mid-plane of the composite structure, while the corresponding values for the bending stiffness matrix $\mathbf{D}$ are symmetric with respect to the mid-plane. See (Reddy, 2004), Tsai and Hahn (1980) and (Gürdal et al., 1999).

A composite structure can experience complex deformation modes due to coupling effects which exist in the stiffness matrices given in equations (9a)-(9d) which arise from the lay-up sequence of the structure. If the lay-up sequence is such that the coupling stiffness matrix $\mathbf{B}$ in equation (7) cannot be neglected, it will give rise to coupling effects between in-plane and bending deformations of the composite structure. These effects can result in a structure experiencing a bending deformation in conjunction to an extensional deformation even though only an in-plane load is applied to the structure. The opposite coupling effect occurs when pure bending is applied instead. Apart from the extensional-bending coupling deformations, a more complex extensional-twisting deformation mode of the composite structure can be induced by the $B_{14}$ and $B_{24}$ elements, without any twisting being applied to the structure. To avoid these deformation modes advantage can be taken of the antisymmetry of the weight factor values for the coupling stiffness matrix $\mathbf{B}$ in equation (9c), by requiring the lay-up sequence to be symmetric with respect to the mid-plane line. This will result in the coupling stiffness matrix $\mathbf{B}=0$, thus eliminating it from equation (7) and uncoupling the in-plane and bending deformations of the structure. However, even with the in-plane and bending deformations uncoupled, there will still exist some coupling effects which arise from the off-axis $\pm \theta$ plies in the lay-up sequence. These are the shear-extension coupling which is induced by the $A_{14}$ and $A_{24}$ elements in equation (9a), the transversal shear coupling induced by the $\tilde{A}_{45}$ in equation (9b) and the bending-twisting coupling induced by the the $D_{14}$ and $D_{24}$ elements in equation (9d). As the extensional stiffness matrix $\mathbf{A}$ in equation (9a) and transversal shear matrix $\tilde{\mathbf{A}}$ in equation (9b) are independent of the lay-up sequence, the shear-extension and transversal shear coupling effects, respectively, can be eliminated by purely requiring the composite structure to contain the same number of off-axis $\pm \theta$ plies. The bending-twisting coupling can however not be entirely eliminated due to the symmetry of the weight factor values of the bending stiffness matrix $\mathbf{D}$, with respect to the mid-plane of the structure, but its effect on the composite structure can be minimized by a correct choice of the lay-up sequence of UD plies. See (Reddy, 2004), Tsai and Hahn (1980) and (Gürdal et al., 1999).

In general the coupling effects are often unwanted in composite structures as they can lead to, for example, delamination of plies in the lay-up sequence or fractures in the fibrous constituents of the plies. The consequence of this is the degradation of the mechanical properties which in turn will affect the mechanical behaviour of the composite structure. Therefore, the minimization of these effects or, if possible, their elimination is often desired when designing composite structures. 



\section{Review of included papers}

\section{Paper I}

\section{Density Filter Control of Thickness-to-Length Change of Composite Structures}

In the first paper, a stiffness optimization problem denoted the homogenization material problem (HMO) is introduced, with the objective to minimize the compliance of a composite structure. The main focus of the paper is to investigate the ability of a linear density filter in controlling the thickness variation of the composite structure. This is investigated by performing a number of numerical tests for different load cases, mesh densities and range of the density filter. The results showed that a density filter can be used to control the thickness variation of a composite structure.

\section{Paper II}

Stiffness and Lay-up Optimization of Composite Structures based on a Homogenized Material Approach

In the second paper, a two phase composite structure optimization method based on a homogenized material approach is presented. Here, both the homogenized material optimization problem (HMO) and the lay-up optimization problem $(\mathrm{LuO})$ formulations of the method are described in detail. To investigate the ability of the proposed method to obtain manufacturable composite structures, a numerical test was performed on a cantilever plate for a number of different parameters settings of the optimization problems and load cases. The result of the test shows that the proposed method can obtain a composite structure with maximized stiffness properties along with a manufacturable lay-up sequence of discrete plies. 



\section{Bibliography}

M.P. Bendsøe and O. Sigmund. Topology optimization: theory, methods, and applications. Springer Verlag, 2003.

Flake C Campbell. Structural Composite Materials. Asm international, 2010.

P.W. Christensen and A. Klarbring. An introduction to structural optimization, volume 153. Springer Verlag, 2008.

RD. Cook, DS. Malkus, ME. Plesha, and RJ. Witt. Concepts and Applications of Finite Element Analysis. John Wiley \& Sons Inc., 4th edition, 2003.

Zafer Gürdal, Raphael T Haftka, and Prabhat Hajela. Design and optimization of laminated composite materials. Wiley.com, 1999.

J.N. Reddy. Mechanics of laminated composite plates and shells: theory and analysis. CRC, 2004.

K. Svanberg. The method of moving asymptotes-a new method for structural optimization. International journal for numerical methods in engineering, 24(2): 359-373, 1987. doi: 10.1002/nme.1620240207.

S.W. Tsai and H.T. Hahn. Introduction to composite materials. CRC Press, 1980.

Laurence A. Wolsey. Integer Programming. Wiley, New York, 1998. 


\section{Part II}

\section{Included Papers}

The articles associated with this thesis have been removed for copyright reasons. For more details about these see:

http://urn.kb.se/resolve?urn=urn:nbn:se:liu:diva-102316 